\title{
Using Filter Bank Multicarrier Signals for Radar Imaging
}

\author{
Sebastian Koslowski, Martin Braun and Friedrich K. Jondral \\ Communications Engineering Lab, Karlsruhe Institute of Technology (KIT), Germany \\ sebastian.koslowski@kit.edu, martin.braun@ettus.com, friedrich.jondral@kit.edu
}

\begin{abstract}
This paper presents an adaption of a joint OFDM communication and radar system to filter bank multicarrier signals. Our results show that any signal which can be represented as a matrix of symbols spread out in the time-frequency domain in a regular fashion can be processed in the same way, making the results obtained from OFDM radar research applicable to FBMC radar. For OFDM, it was shown that the two-dimensional periodogram is a robust and optimal way to obtain a rangeDoppler plane; this method can also be applied to FBMC radar. However, the absence of a cyclic prefix in FBMC modulated signals entails a faster deorthogonalisation of the received symbol for targets with a high range. This is mitigated by the higher processing gain in FBMC radar and the exploitation of the intrinsic interference.
\end{abstract}

Index Terms-OFDM, FBMC, OQAM, Radar, Car-to-car

\section{INTRODUCTION}

Many applications which require radar sensors also benefit from communication capabilities. An example is automotive radar: Vehicles are equipped with radar sensors to detect other traffic participants, avoid collisions and increase road safety and driver assistance. At the same time, car-to-car communication systems may be active to allow vehicles to share their information and further increase safety by cooperation. Another example are unmanned or autonomous vehicles which may already have a communication system and could benefit from radar capabilities to e.g. improve navigation and relative localization of other vehicles in the vicinity [1].

Given the similar nature of radar and wireless communication, it is an obvious question to ask if these components could not be combined into a signal system, which performs radar imaging by analyzing the backscatter from its own communication signal.

One solution suggested multiple times in the last decade is OFDM radar [2]. Here, a transmitter modulates information into an OFDM signal and sends it out for others to receive. This transmitter is connected to a receiver, which shares a clock with the transmitter and thus can synchronously receive the backscattered signal, while others can receive the transmitted signal to demodulate it. Because the transmitter path is the same for the radar and communication signals, this saves both hardware and, since the same signal is used for both purposes, spectrum allocation.

OFDM has been proven to work very well for this kind of application [3], and multiple publications exist which analyze the performance of OFDM radar in various applications and scenarios [4]. OFDM radar does not suffer from the same problems that other radar systems do, e.g., range and Doppler estimation are independent in OFDM radar [5].

OFDM has some disadvantages, though, such as high out-of-band emissions and susceptibility to Doppler spread. For this reason, researchers have suggested communication using filter bank multicarrier (FBMC, also known as OFDM/OQAM) modulation as an alternative to OFDM, in particular for applications where these disadvantages matter, such as vehicular communication. FBMC has been proven to outperform OFDM in some cases [6], [7], [8] for communication purposes.

For the case of combined communication and radar systems, it is therefore an open question if FBMC modulated signals are equally useful for radar processing as OFDM signals. Both systems use a regular-spaced time-frequency grid to spread the transmission symbols over both time and frequency within the assigned duty cycle and allocated bandwidth. Where OFDM uses a guard interval, FBMC employs Nyquist pulse shaping and a modified sub-carrier modulation to ensure orthogonality between symbols and sub-carriers. Assuming frame-based channel access, the transmitted information and the estimates thereof at the output of a respective receiver can be represented as matrix which can be exploited for radar application.

The remainder of this paper is structured as follows: In Section II we describe our system model and briefly review the similarities of OFDM and FBMC transmission schemes. In Section III the proposed radar signal processing for FBMC modulated signals is derived and discussed. In Section IV we show some results obtained by simulation. Finally, Section V concludes.

\section{SYSTEM DESCRIPTION}

The basic system architecture is depicted in Fig. 1. The signal path in the upper half is the transmission chain of the communication part of the system. The information to be transmitted is converted to a base band signal $s(t)$ using either OFDM or FBMC modulation followed by up-conversion in the analog frontend.

In the lower half the signal path for the reception and processing of backscattered signals is depicted. The analog frontend as well as the demodulation are shared between the communication and the radar part of the system. Here, we focus on the radar part which is represented by a divider and the radar processing block following the demodulation. Note that in contrast to a received communication signal, 


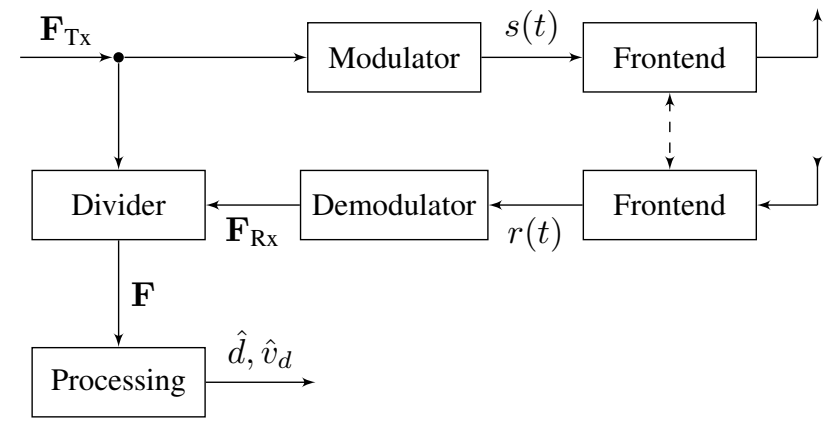

Fig. 1. Joint radar and communication system setup

the data in the incoming signal is known beforehand and no synchronization is performed for the demodulation. However, it is required that both the transmitting and the receiving frontend are synchronized to the same clock.

\section{A. Multicarrier transmission basics}

In order to understand the adaption of OFDM radar processing algorithms for FBMC we will briefly discuss multicarrier transmission and build a common signal model for both schemes. Most multicarrier systems use parallel orthogonal equally-spaced sub-carriers, each linearly modulated with data symbols. The basic transmit signal can be expressed by

$$
s(t)=\sum_{k=0}^{N-1} \sum_{n=-\infty}^{\infty} c_{k, n} g(t-n T) e^{j 2 \pi \Delta f k(t-n T)},
$$

where $T$ is the symbol duration, $N$ the number of carriers, $\Delta f$ the sub-carrier spacing and $g(t)$ the pulse filter impulse response used to modulate the symbols $c_{k, n}$ drawn from a complex modulation alphabet.

In an OFDM system a simple, rectangular pulse of length $T$ is used. The modulated symbols are therefore disjoint in time, but overlap in the frequency domain. Orthogonality between sub-carriers is achieved if $T \geq \Delta f^{-1}$. Using digital signal processing at a sample rate of $f_{s}=\Delta f$, an OFDM signal can efficiently be generated by applying an Inverse Discrete Fourier Transformation (IDFT) of length $N$ to the symbols $c_{k, n}$. In practical systems the symbol duration is chosen to be longer than $\Delta f^{-1}$ resulting in a cyclic prefix $(\mathrm{CP})$ between symbols which is inserted after the IDFT (see Fig. 2). At the receiver the $\mathrm{CP}$ is discarded before the signal is demodulated using a Discrete Fourier Transformation (DFT). The resulting samples $\hat{c}_{n, k}$ are estimates for the transmitted symbols. Note, that using a $\mathrm{CP}$ not only prevents inter-symbol interference, it also makes OFDM, to some extend, robust against signal delays, which is beneficial for radar.

FBMC is a more generalized approach to multicarrier transmission [9]. The basic idea is to use a spectrally confided pulse filter thereby reducing the overlap between sub-carriers and significantly lowering out-of-band emissions. However, orthogonality between the symbols and sub-carriers still has to be maintained. A pulse filter with such properties comes at the price of an extended impulse response length, usually spanning over several symbol periods $T$. Therefore, compared to OFDM, the overlap in the frequency domain is reduced at the expense of the overlap in time [10]. Using a guard interval, like the CP in OFDM, is unfeasible for FBMC systems.

To achieve the desired spectral properties while maintaining a high spectral efficiency requires a relaxation of the orthogonality condition by restricting it to the real component. Consequently, only real-valued symbols can be transmitted without interference despite the use of a pulse filter. To maintain the same data rate, the symbol rate is doubled. Since now the sub-carrier signals have twice the bandwidth, adjacent sub-carriers interfere. By applying a phase offset of $\pi / 2$ between neighboring symbols in both, time and frequency, the required orthogonality is achieved. This scheme is equivalent to an Offset QAM (OQAM) modulation of the sub-carriers: Delaying the imaginary part of the symbols $c_{n, k}$ in (1) by $T / 2$ and applying the aforementioned phase offset between sub-carriers results in the same signal. For this reason, FBMC has alternatively been called OFDM/OQAM [11].

For our purposes the former, real-valued interpretation is more suitable. The FBMC transmit signal is

$$
s(t)=\sum_{k=0}^{N-1} \sum_{n=-\infty}^{\infty} a_{k, n} \theta_{n, k} g(t-n T / 2) e^{j 2 \pi \Delta f k(t-n T / 2)},
$$

where $a_{k, n}$ are Pulse Amplitude Modulation (PAM) symbols with $a_{k, 2 n}=\Re\left(c_{n, k}\right)$ and $a_{k, 2 n+1}=\Im\left(c_{n, k}\right) . \theta_{k, n}=\frac{\pi}{2}(n+k)$ is the additional phase term and $\Delta f=T^{-1}$ resulting in maximal spectral efficiency (given a certain modulation alphabet). In discrete-time, $s(t)$ can be efficiently generated by a TMUX synthesis filter bank with over-sampled inputs [9]. It uses of a bank of poly-phase filters, which jointly modulate the phasecoded transmit symbols $a_{k, n} \theta_{n, k}$ onto the sub-carrier signals followed by an IDFT operation, which shifts the sub-carrier signals to their respective center frequencies in the base-band (see Fig. 2).

The demodulation of FBMC signals is performed analogously to OFDM by performing the inverse transmitter operations, effectively implementing a matched filter per subchannel. The resulting samples are

$$
b_{k, n}=\left.g^{*}(-t) * r(t) e^{-j 2 \pi \Delta f k t}\right|_{t=n T / 2},
$$

where $r(t)$ the received signal. The corresponding discretetime processing uses an analysis filter bank with two-times over-sampled outputs. From the samples $b_{k, n}$ estimates of the transmitted symbols can be obtained by removing the phasecoding and taking the real part:

$$
\hat{a}_{k, n}=\Re\left\{\theta_{n, k}^{*} b_{k, n}\right\} .
$$

As can be seen from (1) and (2) OFDM and FBMC can be described using similar signal models. Also, the required signal processing structures are quite similar as shown in Fig. 2. An OFDM modulation uses straight forward quadrature modulation, whereas FBMC signals are build from phasecoded real-valued symbols with a spacing of $T / 2$. 


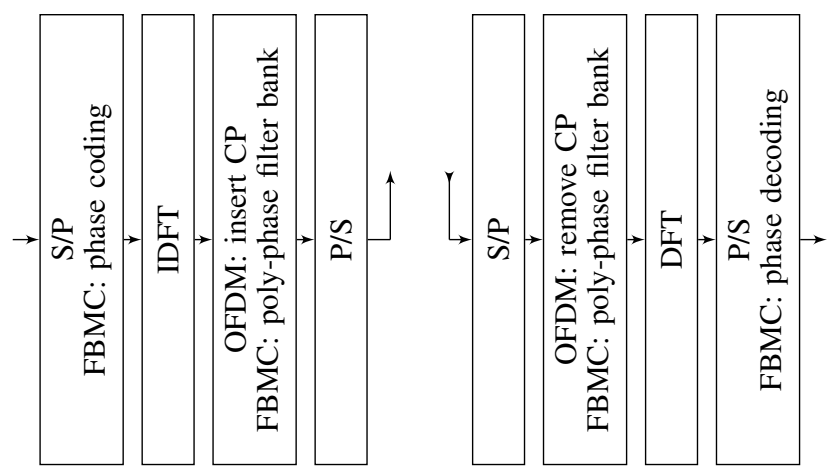

Fig. 2. Modulator and demodulator block diagram for base band OFDM and FBMC signals

\section{OFDM AND FBMC RADAR}

In this section we review the notion of the radar processing matrix which is the common basis for various detection algorithms known for OFDM radar [12, Chap. 3]. Our goal is to find a corresponding matrix representation for FBMC signals making these algorithms directly applicable to FBMC radar.

\section{A. Basic signal model}

In a joint radar and communication system, whether FBMC or OFDM is used, the receiver is active during the transmission of a frame. Backscattered signals reflected from nearby targets are received simultaneously and demodulated as if they originated from another terminal. Each frame consists of $M$ consecutive multicarrier symbols with $N$ active subcarriers. Given a set of signal parameters, a transmitted frame can be represented by a matrix $\mathbf{F}_{\mathrm{Tx}} \in \mathbb{C}^{N \times M}$, where the individual elements $\left(\mathbf{F}_{\mathrm{Tx}}\right)_{k, n}$ are the transmitted modulation symbols $c_{k, n}$. The resulting signal is transmitted, reflected from an object at range $d$ and a relative velocity of $v_{d}$ and finally received. Because the receiver is synchronized to the transmitter rather than the incoming signal, the frame is received with a delay $\tau=2 d / c_{0}$ and frequency shifted by a Doppler shift $f_{D}=f_{C} \frac{v_{r}}{c_{0}}$ with respect to the transmit signal, where $c_{0}$ is the speed of light and $f_{C}$ the center frequency of the signal.

The backscattered signal from each target results in a signal $r(t)=s(t-\tau) e^{j 2 \pi f_{d} t}$ at the receiver. All of the processing is symbol-based, which is why we only consider the demodulator output, represented as a matrix, $\mathbf{F}_{\mathrm{Rx}} \in \mathbb{C}^{N \times M}$. Because the demodulator does not compensate for synchronization errors, the symbol estimates in $\mathbf{F}_{\mathrm{Rx}}$ contain additional phase terms depending on $\tau$ and $f_{D}$. A time delay $\tau$ results in a sub-carrier dependent phase term $e^{-j 2 \pi\left(f_{0}+k \Delta f\right) \tau}$, whereas a frequency shift results in a phase term $e^{j 2 \pi T n f_{D}}$ depending on the symbol index. Assuming a total of $H$ reflections reaching the receiver and ignoring interference terms now (see
Section III-D), the receive matrix elements are

$\left(\mathbf{F}_{\mathrm{Rx}}\right)_{k, l}=\sum_{h=0}^{H-1}\left(\mathbf{F}_{\mathrm{Tx}}\right)_{k, l} \cdot b_{h} e^{j 2 \pi\left(l T_{O} f_{D, h}-k \tau_{h} \Delta f\right)} e^{j \varphi_{h}}+(\mathbf{Z})_{k, l}$

$\tau_{h}$ and $f_{D, h}$ are the individual delays and Doppler shifts, $b_{h}$ the attenuation resulting from the propagation loss to and from the reflecting object as well as its radar cross section (RCS). The phase term $\varphi_{h}$ accounts for all random phase changes and the matrix $\mathbf{Z}$ represents the AWGN after the demodulator. From $\mathbf{F}_{\mathrm{Rx}}$ the $\tau_{h}$ and $f_{D, h}$ have to be estimated. However, before performing any radar processing, the influence of the transmitted data has to be removed; here, we use an elementwise division

$$
(\mathbf{F})_{k, l}=\frac{\left(\mathbf{F}_{\mathrm{Rx}}\right)_{k, l}}{\left(\mathbf{F}_{\mathrm{Tx}}\right)_{k, l}}
$$

resulting in the radar processing matrix $\mathbf{F}$. Ideally it contains sinusoids only: one row- and one column-wise, respectively, for every target.

\section{B. FBMC adaptation}

To apply this technique to FBMC signals, an equivalent matrix $\mathbf{F}$ has be found. Using the complex transmit symbols $c_{n, k}$ as elements in the matrix $\mathbf{F}_{\mathrm{Tx}}$ is not a viable option, because the real and imaginary part are not transmitted at the same time due to the OQAM modulation. The influence of time-varying phase terms such as those caused by a Doppler shift can no longer be described as pure sinusoidal in the matrix $\mathbf{F}_{\mathrm{Rx}}$ of complex symbol estimates $\hat{c}_{n, k}$. That is why, for FBMC we describe a transmit frame using the real-valued PAM symbols from (2). The resulting matrix $\mathbf{F}_{\mathrm{Tx}} \in \mathbb{R}^{N \times 2 M}$ has elements $\left(\mathbf{F}_{\mathrm{Tx}}\right)_{k, n}=a_{k, n}$. Note, that we now have two columns per complex symbol and are therefore able to separately describe phase terms for both, real and imaginary part of each symbol at the receiver.

For the receive matrix $\mathbf{F}_{\mathrm{Rx}}$, the respective real-valued symbol estimates $\hat{a}_{n, k}$ are not very useful as they no longer contain the required phase information. Instead, we omit taking the real part in the demodulator and use the phase-corrected analysis filterbank output, $\left(\mathbf{F}_{\mathrm{Rx}}\right)_{k, n}=\theta_{n, k}^{*} b_{n, k}$. At the receiver, the imaginary part of these samples is generally not zero. The relaxed orthogonality condition in FBMC requires only the real part of symbols not to interfere. As a consequence the imaginary part of each received symbol may be affected by the surrounding symbols under the influence of the pulse shape. This so called intrinsic interference is a result of maximally tight packing of symbols in the time-frequency-plane. In order to remove the modulation symbols from $\mathbf{F}_{\mathrm{Rx}}$ and calculate a radar processing matrix $\mathbf{F}$ it is not sufficient to simply divide by $\mathbf{F}_{\mathrm{Tx}}$.

Since we have the luxury of knowing the transmitted data symbols at the receiver, we can pre-calculate the expected imaginary part of each symbol: Setting $a_{k, n}=\delta_{k} \delta_{n}$ and $r(t)=s(t)$ in (3), thereby connecting the to syntheses and the analysis filterbank back-to-back, results in the transmultiplexer impulse response $A_{\Delta n, \Delta k}^{(k)}$. Table I shows exem- 
TABLE I

TRANSMULTIPLEXER IMPULSE RESPONSE FOR A PHYDYAS FILTER WITH PHASE CODING

\begin{tabular}{|c|c|c|c|c|c|c|c|c|c|}
\hline & $n-4$ & $n-3$ & $n-2$ & $n-1$ & $n+0$ & $n+1$ & $n+2$ & $n+3$ & $n+4$ \\
\hline$k-1$ & $0.005 \mathrm{j}$ & $-0.043 \mathrm{j}$ & $0.125 \mathrm{j}$ & $-0.206 \mathrm{j}$ & $0.239 \mathrm{j}$ & $-0.206 \mathrm{j}$ & $0.125 \mathrm{j}$ & $-0.043 \mathrm{j}$ & $0.005 \mathrm{j}$ \\
\hline$k+0$ & 0 & $0.067 \mathrm{j}$ & 0 & $0.564 \mathrm{j}$ & 1.000 & $-0.564 \mathrm{j}$ & 0 & $-0.067 \mathrm{j}$ & 0 \\
\hline$k+1$ & $-0.005 \mathrm{j}$ & $-0.043 \mathrm{j}$ & $-0.125 \mathrm{j}$ & $-0.206 \mathrm{j}$ & $-0.239 \mathrm{j}$ & $-0.206 \mathrm{j}$ & $-0.125 \mathrm{j}$ & $-0.043 \mathrm{j}$ & $-0.005 \mathrm{j}$ \\
\hline
\end{tabular}

plary values for a PHYDYAS pulse filter [10]. Expect for the coefficient at $(k, n)$ all entries are purely imaginary and thus orthogonal to the transmitted symbol, only contributing to the intrinsic interference. For pulses with good frequency confinement, only the directly adjacent sub-carriers show significant values. The influence the of previous and following symbols is determined by the length of the pulse filter impulse response, here $T_{g}=N K / f_{s}$, where $K=4$ is the number of overlapping symbols. Note that these coefficients generally apply to all symbol positions $(n, k)$. For odd sub-carrier indexes $k$, however, the sign of every other column is flipped. Using the coefficients $A_{\Delta n, \Delta k}^{(k)}$ the modified transmit matrix elements are

$$
\left(\tilde{\mathbf{F}}_{\mathrm{Tx}}\right)_{n, k}=a_{n, k}+\sum_{(\Delta n, \Delta k) \in \Omega} a_{n+\Delta n, k+\Delta k} A_{\Delta n, \Delta k}^{(k)},
$$

where $\Omega$ is the set of symbols positions $(\Delta n, \Delta k)$ contributing to the intrinsic interference. With $\tilde{\mathbf{F}}_{\text {Tx }}$ we can now calculate $\mathbf{F}$ using (6) and proceed with the same radar algorithms known for OFDM radar. The only difference is that there are twice as many points in time, which means that the phase due to a Doppler shift $f_{D, h}$ is halved, and becomes $e^{j \pi T n f_{D}}$.

\section{Periodogram-based estimation}

Here, we briefly outline the algorithm used in the Processing block in Fig. 1. Using a two-dimensional periodogram has proven to be a good solution to estimate the parameters of each pair of sinusoids in the radar processing matrix $\mathbf{F}$; it is defined as

$$
\operatorname{Per}_{\mathbf{F}}(n, m)=\frac{1}{N M}\left|\sum_{k=0}^{N_{\text {Per }}-1} \sum_{l=0}^{M_{\text {Per }}-1}(\mathbf{F})_{k, l} e^{j 2 \pi\left(\frac{k n}{N_{\mathrm{Per}}}-\frac{l m}{M_{\mathrm{Per}}}\right)}\right|^{2} .
$$

where $N_{\text {Per }}$ and $M_{\text {Per }}$ determine the quantization of the resulting frequency plane and are usually a multiples of $N$

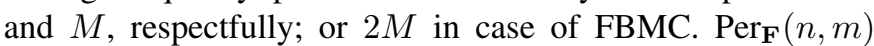
can be efficiently calculated using a combination of FFTs and IFFTs. Combined with a window matrix, e.g. a twodimensional hamming window to minimize side-lobes, this results in a interpolation of $\operatorname{Per}_{\mathbf{F}}(n, m)$ easing the following peak detection A peak at $\hat{n}, \hat{m}$ corresponds to a target at

$$
\hat{d}=\frac{\hat{n} c_{0}}{2 \Delta f N_{\mathrm{Per}}} \quad \text { and } \quad \hat{v}_{D}=\frac{\hat{m} c_{0}}{2 f_{C} T M_{\mathrm{Per}}},
$$

where $T$ is replaced by $T / 2$ for FBMC. Details of this algorithm as well as additional measures to increase the resolution can be found in [12, Chap. 3].

\section{Signal requirements}

In order for (5) to be a valid description of the backscattered signal, the transmitted signal must fulfill certain requirements with respect to the application in mind. Specifically, the basic signal parameters described in Section II-A must be chosen with respect to the expected maximum range and maximum absolute relative velocity of the system.

As for all systems with symbol-based processing, deorthogonalisation must be avoided. Given a set of signal parameters, namely a symbol duration $T$ and a sub-carrier spacing $\Delta f$, the mismatch between the transmit and receiver filters due to delay and frequency shift of the signal must be negligible. Otherwise, the matrix $F$ no longer contains sinusoids only, but also inter-symbol and inter-carrier interference (ISI, ICI) terms. Unlike the intrinsic interference found in FBMC signals, these terms depend not only on the modulation symbols, but also on the target parameters to be estimated. Therefore, they can not be suppressed by applying (6).

To avoid deorthogonalisation in OFDM radar systems it was shown in [5], that the length of the cyclic prefix $T_{G}=T-$ $\Delta f^{-1}$ must be greater than $\tau$ and the maximum Doppler shift must be much smaller than the sub-carrier spacing $\Delta f$. Also, the Doppler shift must be consistent over all carriers, which means $B \ll f_{C}$.

Although the signals of both systems can be described in similar manner, the differences between OFDM and FBMC do carry over into the quality of the radar processing. OFDM guarantees orthogonality between consecutive OFDM symbols by adding a cyclic prefix, thus shielding the OFDM symbols from one another. Except for a higher signal attenuation $b$ backscattered signals are unimpaired from an increasing range. With FBMC modulated signals, this is not the case, and echoes from earlier FBMC symbols, e.g. due to delayed backscattering, will interfere with the following ones. Consequently, the maximum delay must be small in comparison to the symbol duration.

Such a loss in signal-to-interference ratio (SIR) can also be observed in a regular FBMC receiver setup. In an uplink scenario, for example, time and frequency offset corrections are performed after splitting the signal into sub-carrier resulting in a similar model as (5). In [13], [14] it was shown that the SIR degradation due to a frequency shift is less severe for FBMC than for OFDM signals. This translates to a better performance when dealing with targets with high relative velocities. OFDM signals are not susceptible to negative timing offsets (delay) as long they do not exceed the guard interval. Beyond that, however FBMC signals show a similar advantage as seen for 
frequency shifts.

In summary, to get good signal properties for targets with a large range or high relative velocity, a long symbol duration and a big sub-carrier spacing are required. At the same time, a higher number of sub-carriers and symbols per frame beneficial for the radar processing itself. Also, the communication subsystem has almost opposite requirements when it comes to data-rate and robustness against multi-path propagation. Considering the limitation for the overall system bandwidth and frame duration, a compromise has to be found. Because FBMC systems operate without any spectral redundancy, $T$ and $\Delta f$ are directly dependent, which adds another constraint.

\section{Simulations}

To evaluate the proposed adaption of sample-based OFDM radar processing to FBMC modulated signals, a simulation has been implemented in MATLAB. The system is modeled as shown in Fig. 1 where compared to a OFDM radar system the modulator and demodulator have been replaced by their FBMC counterparts (2) and (3). The Division block now also includes the pre-calculation of the expected intrinsic interference (7). Finally, the processing block remains unchanged, although it now operates on a matrix with twice as many columns and a spacing of only $T / 2$ between them.

The signal parameters have been chosen as follows: The center frequency $f_{C}$ is set to $24 \mathrm{GHz}$, the sub-carrier spacing $\Delta f=90.909 \mathrm{kHz}$, and the symbol duration $T=11 \mu \mathrm{s}$ and the aforementioned PHYDYAS filter for FBMC. For OFDM we set $T=12.375 \mu$ s. Frames are comprised of $M=256$ symbols and $N=1024$ QPSK modulated subcarriers carrying random data. The transmit power is set to $25 \mathrm{dBm}$, the receiver is modeled with a noise figure of $5 \mathrm{~dB}$ and both antennas have zero gain.

We place two point-scatterers with an RCS of $10 \mathrm{~m}^{2}$ and $20 \mathrm{~m}^{2}$ at $d_{1}=50 \mathrm{~m}$ and $d_{2}=150 \mathrm{~m}$ with relative velocities $v_{d, 1}=-80 \mathrm{~m} / \mathrm{s}$ and $v_{d, 2}=0 \mathrm{~m} / \mathrm{s}$, respectively. Choosing FBMC as modulation and using the estimator from (8) with $N_{\text {Per }}=4 N$ and $M_{\text {Per }}=8 M$, we can calculate the periodogram shown in Fig. 3. Compared to the result when employing OFDM (in Fig. 4), two effects can observed: First, due to the target at $d_{2}$, the periodogram for FBMC suffers from higher interference than OFDM. Secondly, using FBMC results in higher peaks compared to OFDM. This is the result of the bigger size of $\mathbf{F}$, which increases the processing gain of (8). Also, due to the intrinsic interference and because FBMC uses all of the received signal instead of discarding the $\mathrm{CP}$ of each symbol, the total energy of the matrix $\mathbf{F}$ is higher than the one for OFDM.

Both of these periodograms are the result a single measurement, we now evaluate the signal-to-interference-and-noiseratio (SINR) when averaging over multiple frames. We assume a single target with RCS of $10 \mathrm{~m}^{2}$, varying distance $d_{1}$ and no relative velocity. For each $d_{1}$ we calculate the ratio between the power at the expected bin and the mean of the periodogram excluding the vicinity of that bin. Fig. 5 shows the result of averaging 100 frames for each distance and signal type. Both

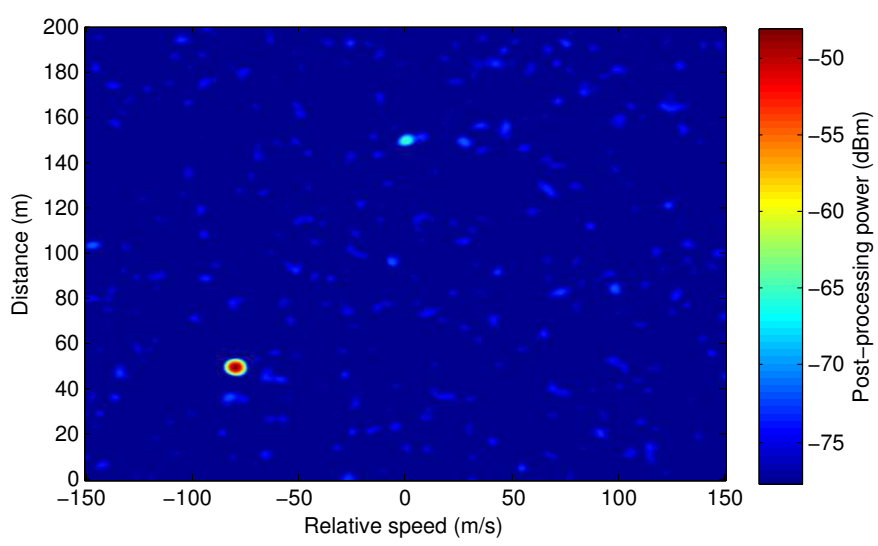

Fig. 3. Periodogram of the radar processing matrix $\mathbf{F}$ derived from the backscatter of a FBMC modulated transmission

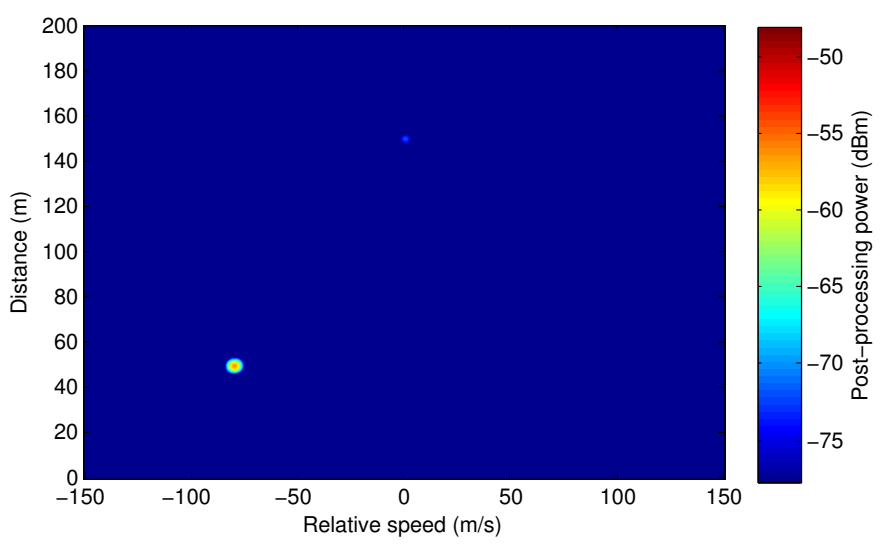

Fig. 4. Periodogram of the radar processing matrix $\mathbf{F}$ derived from the backscatter of a OFDM modulated transmission

systems show almost identical performance which suggests that, at least for the chosen scenario, the added processing gain of FBMC radar equalizes the increased interference due to deorthogonalisation.

\section{CONCLUSION}

In this paper we have investigated an adaption of a combined OFDM communication and radar system for FBMC modulated signals. By finding a similar description for both modulation

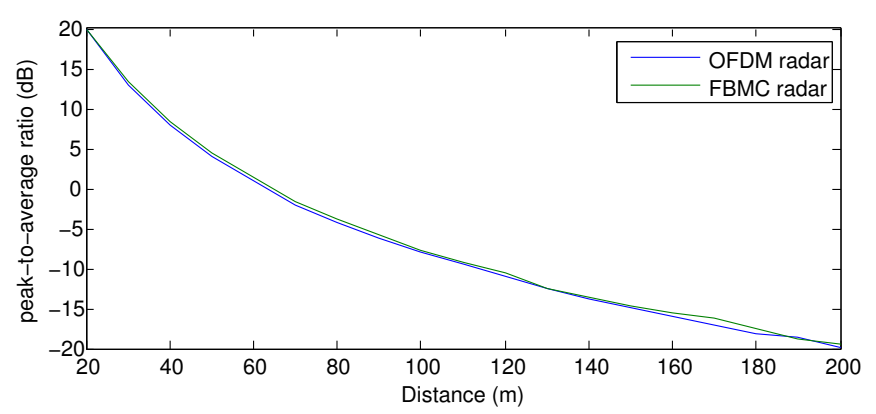

Fig. 5. Comparison of the peak-to-average ratio in the periodogram resulting from a single target scenario 
schemes, we have shown that an analogous signal model of backscattered signals can be formulated. For that, the sampling rate of the demodulator output has to be doubled and the intrinsic interference has to be pre-calculated. All further signal processing and estimation algorithms are the same for both modulation schemes.

Regarding performance, we have discussed the influence of the various signal parameters as well as those of the application scenario. Considering only the radar detection performance, OFDM signals have the great benefit of achieving a higher interference-free range as there is no immediate deorthogonalisation when a CP is employed. On the other hand, using FBMC modulated signals results in a higher processing gain mitigating some of the effects of deorthogonalisation.

This shows that FBMC signals can be used for radar imaging. Communication systems using FBMC can easily be equipped with radar capabilities without the need of additional hardware.

\section{REFERENCES}

[1] A. Benslimane, "localization in vehicular ad hoc networks," in Systems Communications, 2005. Proceedings.

[2] C. Sturm and W. Wiesbeck, "Waveform Design and Signal Processing Aspects for Fusion of Wireless Communications and Radar Sensing," Proceedings of the IEEE, vol. 99, no. 7, pp. 1236-1259, July 2011.

[3] C. Sturm, M. Braun, T. Zwick, and W. Wiesbeck, "Performance Verification of Symbol-Based OFDM Radar Processing," Radar Conference, IEEE International, 2010.

[4] M. Braun, C. Sturm, and F. K. Jondral, "On the Single-Target Accuracy of OFDM Radar Algorithms," 22nd IEEE Symposium on Personal Indoor and Mobile Radio Communications, 2011.

[5] — - "Maximum Likelihood Speed and Distance Estimation for OFDM Radar," Radar Conference, IEEE International, 2010.

[6] S. Premnath, D. Wasden, S. Kasera, N. Patwari, and B. FarhangBoroujeny, "Beyond OFDM: Best-Effort Dynamic Spectrum Access Using Filterbank Multicarrier," Networking, IEEE/ACM Transactions on, vol. 21, no. 3, pp. 869-882, June 2013.

[7] F. Schaich, "Filterbank based multi carrier transmission (FBMC); evolving OFDM: FBMC in the context of WiMAX," in Wireless Conference (EW), 2010 European, April 2010, pp. 1051-1058.

[8] T. Ihalainen, T. Stitz, A. Viholainen, and M. Renfors, "Performance comparison of LDPC-coded FBMC and CP-OFDM in beyond $3 \mathrm{G}$ context," in Circuits and Systems, 2006. ISCAS 2006. Proceedings. 2006 IEEE International Symposium on, May 2006, pp. 4 pp.-.

[9] B. Farhang-Boroujeny, "OFDM Versus Filter Bank Multicarrier," Signal Processing Magazine, IEEE, vol. 28, no. 3, pp. 92-112, May 2011.

[10] A. Sahin, I. Güvenç, and H. Arslan, "A Survey on Prototype Filter Design for Filter Bank Based Multicarrier Communications," CoRR, vol. abs/1212.3374, 2012.

[11] B. Hirosaki, "An Orthogonally Multiplexed QAM System Using the Discrete Fourier Transform," Communications, IEEE Transactions on, vol. 29, no. 7, pp. 982-989, Jul 1981.

[12] M. Braun, "OFDM Radar Algorithms in Mobile Communication Networks," Ph.D. dissertation, Karlsruhe Institute of Technology, 2014, available online at www.cel.kit.edu.

[13] A. Sahin, I. Guvenc, and H. Arslan, "A comparative study of FBMC prototype filters in doubly dispersive channels," in Globecom Workshops (GC Wkshps), 2012 IEEE, Dec 2012, pp. 197-203.

[14] H. Saeedi-Sourck, Y. Wu, J. W. Bergmans, S. Sadri, and B. FarhangBoroujeny, "Sensitivity analysis of offset $\{$ QAM $\}$ multicarrier systems to residual carrier frequency and timing offsets," Signal Processing, vol. 91, no. 7, pp. $1604-1612,2011$. 